\title{
The Robustness of Generalized Estimating Equations for Association Tests in Extended Family Data
}

\author{
Bhoom Suktitipat $^{a, c, d}$ Rasika A. Mathias ${ }^{b}$ Dhananjay Vaidya $^{b}$ Lisa R. Yanek ${ }^{b}$ \\ J. Hunter Young ${ }^{b}$ Lewis C. Becker ${ }^{b}$ Diane M. Becker ${ }^{b} \quad$ Alexander F. Wilson $^{a}$ \\ M. Daniele Fallin ${ }^{b, c}$ \\ ${ }^{a}$ Genometrics Section, Inherited Disease Research Branch, National Human Genome Research Institute, \\ National Institutes of Health, ${ }^{b}$ Department of Medicine, Johns Hopkins Medical Institutions, and \\ 'Department of Epidemiology, Johns Hopkins Bloomberg School of Public Health, Baltimore, Md., USA; \\ ${ }^{\mathrm{d}}$ Department of Biochemistry, Faculty of Medicine Siriraj Hospital, Mahidol University, Bangkok, Thailand
}

\section{Key Words}

Generalized estimating equation • Variance components analysis · Family-based association study $\cdot$ Genome-wide scan

\begin{abstract}
Variance components analysis (VCA), the traditional method for handling correlations within families in genetic association studies, is computationally intensive for genome-wide analyses, and the computational burden of VCA increases with family size and the number of genetic markers. Alternative approaches that do not require the computation of familial correlations are preferable, provided that they do not inflate type I error or decrease power. We performed a simulation study to evaluate practical alternatives to VCA that use regression with generalized estimating equations (GEE) in extended family data. We compared the properties of linear regression with GEE applied to an entire extended family structure (GEE-EXT) and GEE applied to nuclear family structures split from these extended families (GEE-SPL) to variance components likelihood-based methods (FastAssoc). GEE-EXT was evaluated with and without robust variance es-
\end{abstract}

timators to estimate the standard errors. We observed similar average type I error rates from GEE-EXT and FastAssoc compared to GEE-SPL. Type I error rates for the GEE-EXT method with a robust variance estimator were marginally higher than the nominal rate when the minor allele frequency (MAF) was $<0.1$, but were close to the nominal rate when the MAF was $\geq 0.2$. All methods gave consistent effect estimates and had similar power. In summary, the GEE framework with the robust variance estimator, the computationally fastest and least data management-intensive approach, appears to work well in extended families and thus provides a reasonable alternative to full variance components approaches for extended pedigrees in a genome-wide association study setting.

Copyright $\odot 2012$ S. Karger AG, Basel

\section{Introduction}

Genetic association studies in family data are complicated by the non-independence of family members. Special attention is required to handle these within-family correlations. When nuclear families are used, the tests of

\section{KARGER \\ Fax +4161306 1234 \\ E-Mail karger@karger.ch}

www.karger.com
(C) 2012 S. Karger AG, Basel

0001-5652/12/0741-0017\$38.00/0

Accessible online at:

www.karger.com/hhe
M. Daniele Fallin, $\mathrm{PhD}$

615 N. Wolfe Street

Room 6509

Baltimore, MD 21205 (USA)

Tel. +1 410955 3643, E-Mail dfallin@ @ jhsph.edu 
association are straightforward. The child's genotype and phenotype data are analyzed conditioning on the parental genotypes (e.g. conditional logistic regression, transmission disequilibrium test (TDT) [1] and TDT-variant tests, e.g. FBAT [2] and PBAT [3]). These methods do not use the phenotypic information of the parents, even when it is available. When such data do exist, especially in extended pedigrees, methods, such as the variance components analysis (VCA), that incorporate the phenotypic information of the parents to test for association can yield more power. However, modeling the genotype and phenotype data from all family members and allowing for the familial correlation requires likelihood-based methods such as VCA. VCA is widely accepted as the most powerful method for association tests with genotype and phenotype information in a family-based study. However, for large families with a large number of markers, VCA can be computationally intractable. Millions of SNPs used in genome-wide association studies (GWAS) limit the analysis of extended families with full-likelihood methods because of the number of genotype vectors and genotype correlations that must be handled. To facilitate analysis of extended pedigree data, family-based studies with extended families are often split into smaller nuclear families for analysis, losing some genetic information about relationships across those nuclear families.

To address the computation issue, a score version of VCA has been proposed as a computationally efficient alternative to computing the familial likelihood [4]. However, to properly correct for correlation within families, VCA still requires the estimation of the identity-bydescent (IBD) allele-sharing matrix, which is extremely time-consuming. Other variations of VCA have been proposed, such as the Genome-wide Rapid Association using Mixed Model and Regression (GRAMMAR) method [5], a compressed mixed linear model (TASSEL) [6], and the recent Efficient Mixed-Model Association (EMMA and EMMAX) [7, 8]. These methods optimize the IBD-sharing matrix calculation and offer some computational improvement to VCA. Using information based on genetic markers to adjust for correlation allows these methods to properly control for the type I error rate. However, even an efficient algorithm calculating the relationship from genetic markers is still computationally complex.

To avoid computational complexity from modeling relationships based on genetic markers, a simpler correlation model can be used with a linear regression and generalized estimating equations (GEE) $[9,10]$. GEE is a faster method that can accommodate correlation within families and has been previously used in GWAS [11]. GEE accommodates familial clustering by assuming an empirical correlation structure rather than calculating a family-specific correlation, and a robust variance estimator is used in conjunction with GEE to handle the misspecified correlation structure. However, the variance of the association parameters tends to be underestimated, and the type I error rate may be increased [12].

One explanation for an inflated type I error rate could be the misspecification of the correlation structure (all types of member pairs are typically given the same correlation), which makes GEE less efficient [10]. Splitting extended families into nuclear families may help to homogenize the pairwise correlations within a cluster for GEE and thus might improve the efficiency of GEE in family-based association tests. However, in the genomewide association study setting, splitting the data into nuclear families requires additional laborious data manipulation and additional storage space. Although many software packages have been developed to handle this task, it is not a trivial step before the association analysis can be carried out. Since the original data were collected as 'extended pedigree' format, it is easier to analyze the data as they are, i.e. in 'extended pedigree' format, instead of splitting the data into nuclear families. Furthermore, it is possible that splitting extended families into nuclear families could introduce additional correlation among newly generated nuclear families.

Thus, researchers with both phenotype and genotype data on extended families planning to carry out GWAS are faced with practical considerations regarding analyst and computer time and seek an approach that is fast and efficient while accommodating family correlations and maintaining appropriate test size and power. Here, we compare relatively fast GEE methods to variance components methods to examine the properties of each and the time required under a set of scenarios modeled after a practical example in our applied research.

\section{Methods}

We used computer simulation methods to compare the type I error rates and power to detect associations for analyses of family data using: (1) simple linear regression (LM), a method that does not accommodate familial correlations as a baseline; (2) GEE, with clusters based on extended family structure (GEE-EXT); (3) GEE with clusters based on nuclear families derived from splitting original extended family structures (GEE-SPL), and (4) a score test based on VCA (FastAssoc). A total of 10,000 replicates were tested for each pair of genotype-phenotype associations. 


\section{Family Structures}

GeneSTAR Extended Families

The family structure was based on families from the Johns Hopkins GeneSTAR study [13], with the intent of applying these approaches to GWAS applications in this data set. The study is comprised of 3,505 individuals in 498 families, with 242 twogeneration families $(22.1 \%)$ and 260 three-generation families (77.9\%). On average, there were 6.98 members per extended family (ranging from 3 to 26).

Splitting Extended Families into Nuclear Families

We split extended families into nuclear families by duplicating 525 individuals from 260 three-generation families to form 964 nuclear families. We maintained the number of people with genotype and phenotype data by removing phenotypes of duplicated individuals. After splitting extended families, we reduced the number of relative pairs with complete data, i.e. pairs of spouses (families from participants with more than one spouse were split), parent-offspring pairs, and distantly related relatives, but maintained the number of sibling-sibling pairs within nuclear families (table 1). The resulting nuclear families had an average of 3.93 members (ranging from 3 to 12).

\section{Simulated Independent Nuclear Families}

The nuclear families resulting from a split of a single extended family are potentially correlated. Therefore, to compare a data set with a fixed number of nuclear families derived from splitting to one with the same number of nuclear families where all are independent, we simulated independent sets of nuclear families that were completely unrelated to each other. The simulated nuclear families had homogeneous structures with modest size consisting of two parents and four children. A total of 584 families were simulated, so that we had a total of 3,504 individuals in the full data set, similar to the number in our other simulations above.

We then compared type I error rates from the analyses based on nuclear families split from extended families to type I error rates for simulated independent nuclear families of the same size and total numbers.

\section{Genotype Data}

For each simulation, we randomly generated 4 independent biallelic markers with minor allele frequencies (MAF) of 0.05 , $0.10,0.20$, and 0.40 in the founders. A gene-dropping algorithm was implemented in $\mathrm{R}$ version 2.9 [14] to assign genotypes in the offspring recursively, based on Mendelian expectations. These markers were used as input genotype files for subsequent simulations of phenotype relationships.

\section{Simulation of Genotype-Phenotype Association}

We used MERLIN to simulate phenotype-genotype associations in family data $[4,15]$. For each genotype-phenotype association, we simulated 7 phenotypes with locus-specific heritabilities of $0,0.01,0.05,0.1,0.5,1$, and $2 \%$ to mimic heritability levels of complex genetic diseases consisting of both polygenic models and major gene models [16]. All phenotypes were simulated to have a trait heritability of $40 \%$.

\section{Effect of Missing Genotypes}

Because most studies have missing information for some family members, we also explored the effect of missing data on type I
Table 1. Numbers of relative pairs with complete data before and after splitting extended families into nuclear families

\begin{tabular}{llc}
\hline & $\begin{array}{l}\text { Extended families } \\
\text { (502 families) }\end{array}$ & $\begin{array}{l}\text { Nuclear families } \\
(1,206 \text { families })\end{array}$ \\
\hline Parent-offspring & 3,956 & 3,129 \\
Sibling-sibling & 1,606 & 1,606 \\
Grandparent & 1,454 & - \\
Avuncular & 1,482 & - \\
Cousin & 726 & - \\
Spouse-spouse & 1,026 & 506 \\
\hline Individuals & 3,505 & 3,505 \\
\hline
\end{tabular}

error rates for GEE compared to FastAssoc. In the simulated independent nuclear families, we randomly generated 6 levels of missing genotype data: complete data (COMP), missing one parent (M1P), missing both parents (M2P), missing one parent and one child (M1P1C), missing both parents and one child (M2P1C), and missing two parents and two children (M2P2C).

\section{Tests of Association}

We tested for association between each genotype-phenotype pair using (1) LM, (2) GEE-EXT, (3) GEE-SPL, and (4) FastAssoc. We denote the phenotype $y$ and the genotype $g$ for each observation $\left(y_{i j}, g_{i j}\right)$ for member $j$, where $j=1, \ldots, k \ldots, n_{i}$, of family $i$, where $i=1, \ldots, N$.

\section{Linear Regression}

LM was chosen as a test of association to provide a reference as we expect that ignoring correlation within a family would inflate the type I error rate. LM tests for genetic association at each SNP $g_{i j}$ by modeling the mean phenotype value as $\mu_{i j}=E\left(Y_{i j}\right)=$ $\mu+\beta g_{i j}+\varepsilon_{i j}$, where $\mu$ is the population mean. $\beta$ is the additive effect for each $g_{i j}$ SNP, which is coded as 0,1 , or 2 . All observations were treated as if they were independent, regardless of the fact that they are correlated within families, i.e. assuming $\operatorname{Corr}\left(\varepsilon_{i j}, \varepsilon_{i k}\right)=0$. The information concerning their relationship within each family is discarded, leading to a biased estimate of the variance of the regression parameter estimates and the test statistics $[9,10]$.

\section{Generalized Estimating Equations}

GEE were proposed as an extension of the generalized linear modeling to handle clustered data based on a quasi-likelihood approach $[9,10]$. For our genetic association tests, we used the identity link function and modeled the mean phenotypic value conditional on the genotype $g_{i j}$ as $\mu_{i j}=E\left(Y_{i j} \mid g_{i j}\right)=g_{i j}^{\prime} \beta$, with an exchangeable working correlation matrix $\operatorname{Corr}\left(Y_{i j}, Y_{i k}\right)=\alpha$, for all $i$ and $j \neq$ $k$ to accommodate correlation among family members. Robust variance estimators were used to estimate the standard errors of the test statistics to improve the robustness to the model specification error $[17,18]$. 
Table 2. Type I error rate for each association test

\begin{tabular}{lllll}
\hline Critical values* $^{*}$ & \multicolumn{2}{l}{ MAF } & & \\
\cline { 2 - 5 } & 0.05 & 0.10 & 0.20 & 0.40 \\
\hline 0.05 & & & & \\
LM & 0.093 & 0.092 & 0.093 & 0.095 \\
GEE-EXT & 0.053 & 0.053 & 0.051 & 0.049 \\
GEE-SPL & 0.061 & 0.058 & 0.058 & 0.057 \\
FastAssoc & 0.048 & 0.050 & 0.052 & 0.050 \\
0.01 & & & & \\
LM & 0.029 & 0.027 & 0.029 & 0.025 \\
GEE-EXT & 0.012 & 0.012 & 0.013 & 0.011 \\
GEE-SPL & 0.013 & 0.012 & 0.014 & 0.014 \\
FastAssoc & 0.010 & 0.010 & 0.011 & 0.012 \\
0.001 & & & & \\
LM & 0.0040 & 0.0030 & 0.0060 & 0.0040 \\
GEE-EXT & 0.0009 & 0.0009 & 0.0011 & 0.0011 \\
GEE-SPL & 0.0011 & 0.0010 & 0.0013 & 0.0020 \\
FastAssoc & 0.0050 & 0.0070 & 0.0090 & 0.0014 \\
\hline
\end{tabular}

* Type I error rate was calculated as the proportion of the replicates under the null hypothesis with test of association p-values below this critical value.

\section{GEE-EXT and GEE-SPL}

We explored the effect of the family structure in defining a correlation structure: a cluster based on extended families versus a cluster based on nuclear families resulting from splits of extended families. In the case of extended family structures, there are many relative types among family members ranging from closely related, e.g. parent-offspring and sibling-sibling, distantly related, e.g. cousin and avuncular, to unrelated, e.g. spousal relationships, with the expected genetic correlations of $1 / 2,1 / 2,1 / 8,1 / 8$, and 0 , respectively. In nuclear families, there are only three types of familial correlation: parent-offspring, spouse, and sibling-sibling, with the expected genetic correlations of $1 / 2,0$, and $1 / 2$. Therefore, the exchangeable correlation structure is more appropriate for nuclear families than for extended families.

\section{GEE-NR}

To explore the effects of the robust variance estimator on the type I error rate for GEE-EXT, we compared the type I error rate and power for GEE-EXT when using a non-robust standard variance estimator.

\section{Variance Components Analysis}

We used a score version of VCA for testing genetic associations in extended family data $[4,15]$. Briefly, the expected phenotype $E\left(Y_{i j}\right)$ is modeled as $E\left(Y_{i j}\right)=\mu+\beta_{g} \bar{g}_{i j}+\varepsilon_{i j}$, where $\mu$ is the population mean, while $\beta_{g}$ is the additive effect to be estimated for each SNP. The expected genotype score $\bar{g}_{i j}$ is imputed based on the information from the observed neighbor genotype when $g_{i j}$ is missing. The corresponding variance-covariance matrix for family $i$,
$\Omega_{i j k}$, which allows for correlations of phenotypes for all specific types of relative pairs in a family, is defined as

$$
\Omega_{i j k}=\left\{\begin{array}{cc}
\sigma_{a}^{2}+\sigma_{g}^{2}+\sigma_{e}^{2} & \text { if } \mathrm{j}=\mathrm{k} \\
\pi_{i j k} \sigma_{a}^{2}+2 \varphi_{i j k} \sigma_{g}^{2} & \text { if } \mathrm{j} \neq \mathrm{k}
\end{array}\right.
$$

where $\pi_{i j k}$ represents IBD allele sharing, and $\varphi_{i j k}$ represents the kinship coefficient. Conventionally, the expected phenotype and the covariance matrix are used to maximize the multivariate normal likelihood,

$$
L=\prod_{i}(2 \pi)^{-n_{i} / 2}\left|\Omega_{i}\right|^{-1 / 2} e^{1 / 2\left[y_{i}-E\left(y_{i}\right)\right]^{\prime} \Omega_{i}^{-1}\left[y_{i}-E\left(y_{i}\right)\right]},
$$

with and without $\sigma_{a}^{2}$ and $\beta_{g}$. The likelihood ratio test is then used as a test of association for each SNP. To speed up the test for association, $E\left(y_{i}\right)^{\text {base }}$ and $\Omega_{i}^{(\text {base })}$ are estimated once without $\sigma_{a}^{2}$ and $\beta_{g}$ to form a simplified variance components model. Then, an alternate test of association is calculated as

$$
T^{\text {score }}=\frac{\left\{\sum_{i}\left[\bar{g}_{i}-E\left(\bar{g}_{i}\right)\right]^{\prime}\left[\Omega_{i}^{(\text {base })}\right]^{-1}\left[y_{i}-E\left(y_{i}\right)^{(\text {base })}\right]\right\}^{2}}{\sum_{i}\left[\bar{g}_{i}-E\left(\bar{g}_{i}\right)\right]^{\prime}\left[\Omega_{i}^{(\text {base })}\right]^{-1}\left[\bar{g}_{i}-E\left(\bar{g}_{i}\right)\right]}
$$

which can be compared to a $\chi^{2}$ with $1 \mathrm{df}$.

Analyses using LM and GEE were done in R version 2.9 using the functions $\operatorname{lm}()$ in the stats package [14] and gee() in gee package [19]. VCA was done in MERLIN using the fastAssoc option [4, $15]$.

\section{Estimation of Type I Error Rate and Power}

We calculated type I error rates as the proportion of replicates under the null hypothesis (locus-specific heritability set to 0 ) with an association test $p$ value $\leq 0.001,0.01$, and 0.05 . For estimates of power at the $\alpha=0.05$ level, we first adjusted the appropriate type I error critical value for each testing method based on null simulations, and then used the value at which the type I error rate was $5 \%$ as the significance threshold for association. Power was then calculated as the proportion of replicates under a particular alternative that had $\mathrm{p}$ values less than this empirically derived critical value.

\section{Results}

\section{Type I Error Rate}

Across the range of simulated allele frequencies, LM, which ignored the correlation among family members, had inflated type I error rates as expected (table 2). In contrast, VCA had type I error rates equal to the nominal type I error rate across the range of allele frequencies. Type I error rates for the GEE-EXT method (GEE clustered based on the original extended family structure) were slightly higher than the nominal type I error rate when the MAF was $<0.10$, but were close to the nominal rate when the MAF was $\geq 0.20$. GEE-EXT showed type I error rates similar to FastAssoc in most situations. The 
Table 3. The effect of family structure on the type I error rate of GEE

\begin{tabular}{llll}
\hline MAF & $\begin{array}{l}\text { Independent } \\
\text { nuclear families* }\end{array}$ & $\begin{array}{l}\text { Derived split } \\
\text { nuclear families }\end{array}$ & $\begin{array}{l}\text { Extended } \\
\text { families }^{\ddagger}\end{array}$ \\
\hline 0.05 & 0.050 & 0.061 & 0.053 \\
0.10 & 0.052 & 0.058 & 0.053 \\
0.20 & 0.053 & 0.058 & 0.051 \\
0.40 & 0.050 & 0.057 & 0.049 \\
\hline
\end{tabular}

* Simulated nuclear families with no correlation among families. ${ }^{\dagger}$ Splits of the original GeneSTAR extended families into nuclear families. ${ }^{\ddagger}$ Original GeneSTAR extended family structure.

Table 4. Critical values corresponding to a 5\% type I error rate for each method

\begin{tabular}{|c|c|c|c|c|}
\hline & \multicolumn{4}{|l|}{ MAF } \\
\hline & 0.05 & 0.10 & 0.20 & 0.40 \\
\hline LM & 0.023 & 0.022 & 0.021 & 0.022 \\
\hline GEE-EXT & 0.047 & 0.047 & 0.049 & 0.052 \\
\hline GEE-SPL & 0.041 & 0.042 & 0.040 & 0.043 \\
\hline FastAssoc & 0.052 & 0.050 & 0.048 & 0.051 \\
\hline
\end{tabular}

type I error rate for the GEE-SPL method (GEE clustered based on nuclear families resulting from splits of extended families) was slightly inflated. The type I error from GEE applied to the simulated data of independent nuclear families with the same number of individuals, however, yielded type I error rates equal to the nominal type I error rate across the range of allele frequencies (table 3 ). Thus, it appears that correlation across nuclear families introduced by splitting extended families inflates the type I error for GEE.

\section{Power}

To estimate the power, we assumed a fixed total heritability of $40 \%$ while varying the locus-specific heritability from 0.01 to $2 \%$. To account for the effect of inflated type I error rates for some of the methods, we used a corrected threshold to maintain a 5\% type I error when assessing power (table 4). All methods yielded very similar power, although the power from VCA was slightly higher than that from the other methods (fig. 1; online suppl. table 1; for all online suppl. material, see www. karger.com/doi/10.1159/000341636).

Robustness of GEE in Extended Family Data
Table 5. Percent coverage of true effect sizes (using 95\% CI calculated as $\beta \pm 1.96 \times$ S.E.) for each method based on 10,000 replicates

\begin{tabular}{lllllll}
\hline MAF & LH $^{2 *}$ & Beta $^{\dagger}$ & LM & GEE-EXT & GEE-SPL & FastAssoc \\
\hline 0.05 & 0.0001 & 0.032 & 90.63 & 94.54 & 94.05 & 95.20 \\
0.10 & 0.0001 & 0.024 & 90.79 & 94.81 & 94.07 & 94.99 \\
0.20 & 0.0001 & 0.018 & 91.09 & 94.92 & 94.50 & 95.44 \\
0.40 & 0.0001 & 0.014 & 90.71 & 94.67 & 93.91 & 94.90 \\
0.05 & 0.0005 & 0.073 & 91.02 & 94.77 & 93.95 & 95.27 \\
0.10 & 0.0005 & 0.053 & 90.53 & 94.63 & 93.82 & 94.87 \\
0.20 & 0.0005 & 0.040 & 90.67 & 94.83 & 94.00 & 95.12 \\
0.40 & 0.0005 & 0.032 & 90.92 & 95.05 & 94.41 & 95.00 \\
0.05 & 0.0010 & 0.103 & 90.92 & 94.76 & 94.13 & 95.02 \\
0.10 & 0.0010 & 0.075 & 90.77 & 94.99 & 93.99 & 94.76 \\
0.20 & 0.0010 & 0.056 & 91.05 & 95.23 & 94.51 & 95.24 \\
0.40 & 0.0010 & 0.046 & 90.44 & 94.68 & 93.97 & 94.66 \\
0.05 & 0.0050 & 0.229 & 90.50 & 94.22 & 93.45 & 94.64 \\
0.10 & 0.0050 & 0.167 & 90.57 & 94.52 & 93.96 & 94.80 \\
0.20 & 0.0050 & 0.125 & 90.45 & 94.79 & 94.43 & 95.07 \\
0.40 & 0.0050 & 0.102 & 91.19 & 94.77 & 94.30 & 94.81 \\
0.05 & 0.0100 & 0.324 & 90.24 & 94.25 & 93.70 & 94.98 \\
0.10 & 0.0100 & 0.236 & 90.18 & 93.83 & 93.66 & 94.49 \\
0.20 & 0.0100 & 0.177 & 90.66 & 95.02 & 94.24 & 95.26 \\
0.40 & 0.0100 & 0.144 & 90.32 & 94.49 & 93.99 & 94.80 \\
0.05 & 0.0200 & 0.459 & 89.46 & 93.25 & 92.75 & 94.26 \\
0.10 & 0.0200 & 0.333 & 90.39 & 94.52 & 93.87 & 94.79 \\
0.20 & 0.0200 & 0.250 & 91.20 & 94.91 & 94.34 & 95.18 \\
0.40 & 0.0200 & 0.204 & 90.56 & 94.56 & 94.22 & 95.15 \\
\hline Average & & & 90.24 & 94.01 & 93.45 & 94.66 \\
\hline & & & & & \\
\hline
\end{tabular}

* Locus-specific heritability. ${ }^{\dagger}$ Specified effect size used for simulation of the phenotype.

\section{Precision}

The estimated effect sizes from each method were highly correlated and nearly identical (Pearson's correlation $\geq 0.995$; fig. 2). Moreover, the $95 \% \mathrm{CI}$ of the estimated effect size $(\hat{\beta} \pm 1.96 \times$ S.E. $)$ covered the simulated effect sizes $94.7,94.0,93.5$, and $90.2 \%$ of the times for FastAssoc, GEE-EXT, GEE-SPL, and LM, respectively (table 5). Using FastAssoc for comparison, GEE-EXT tended to slightly overestimate the standard errors, whereas GEE-SPL yielded standard errors that were too small (fig. 3). As expected, the standard error from LM was underestimated; hence, the test statistic was overestimated and had a higher type I error rate.

\section{The Effect of Missing Genotypes on Type I Error}

To examine the effect of missing data in nuclear families, we varied the level of missing data from 1 to 4 persons

Hum Hered 2012;74:17-26 


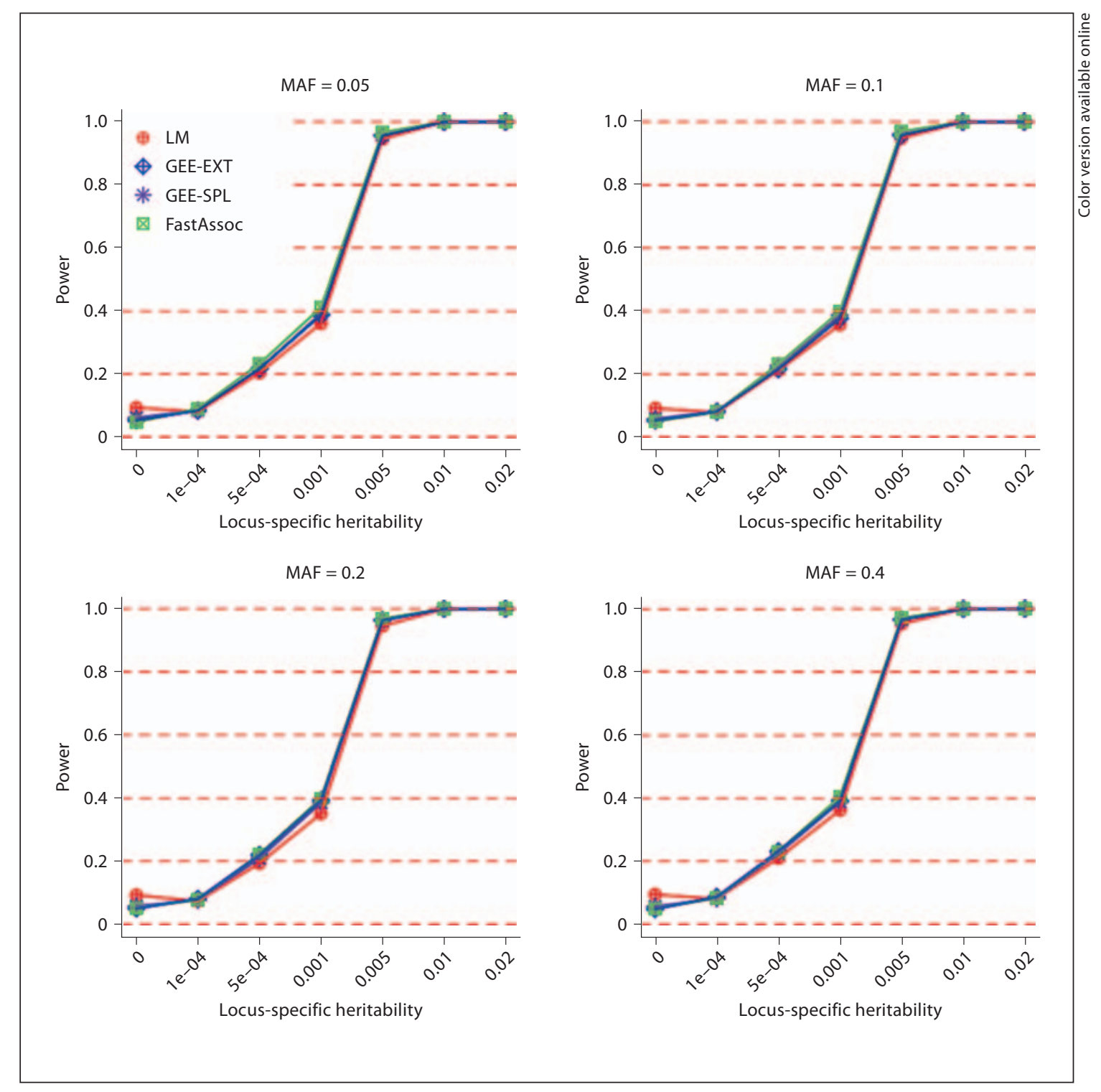

Fig. 1. Estimated power for LM, GEE-EXT, GEE-SPL, and FastAssoc. Each plot shows the estimated power for a MAF of $0.05,0.10,0.20$, and 0.40 , respectively. The $\mathrm{x}$-axis shows the locus-specific heritability used to simulate the phenotype. The total heritability of the phenotype was set to $40 \%$. The power was estimated using a corrected $\mathrm{p}$ value threshold, where $5 \%$ of the signals were detected under the null hypothesis.

out of 6 in a family. We observed that missing data did not affect type I error rates for GEE and FastAssoc, and that these two methods had stable type I error rates across the range of missing data patterns (fig. 4). In contrast, type I error rates observed for LM were lower as the number of missing people per family was higher and the correlation among family members became less relevant (results not shown).

\section{Discussion}

Extended families are a computational problem for GWAS analysis of family data, particularly in studies with extensive phenotyping. Instead of using a computationally intensive variance components method to test for genomewide association signals, we proposed to use generalized linear models with GEE, which require much less computing time while still accounting for correlation within fam- 
Fig. 2. Correlation among effect estimates across association test methods. The numbers in the upper diagonal matrix indicate Pearson correlation coefficients. ${ }^{* *} \mathrm{p}<$ 0.0001 .
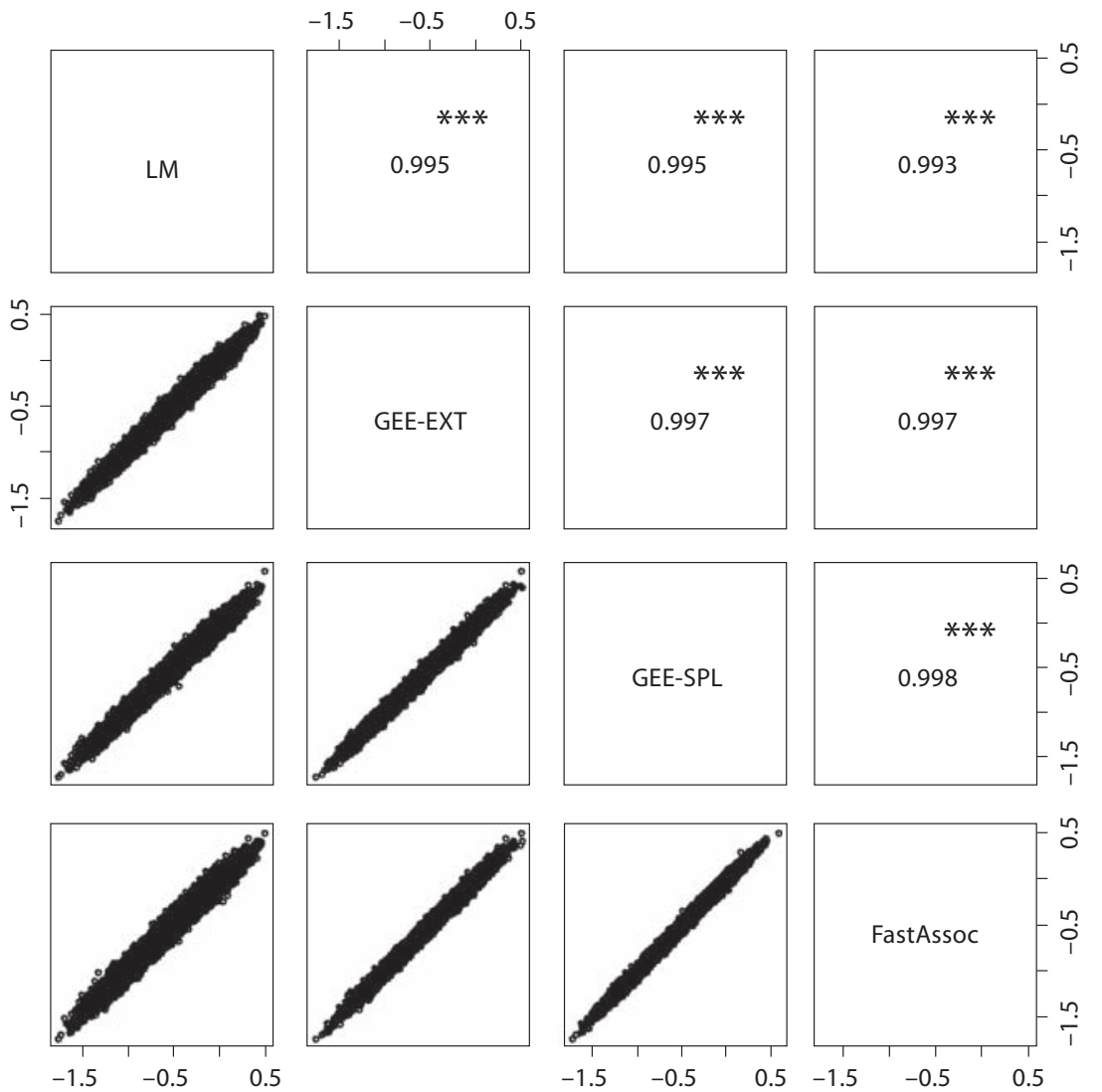

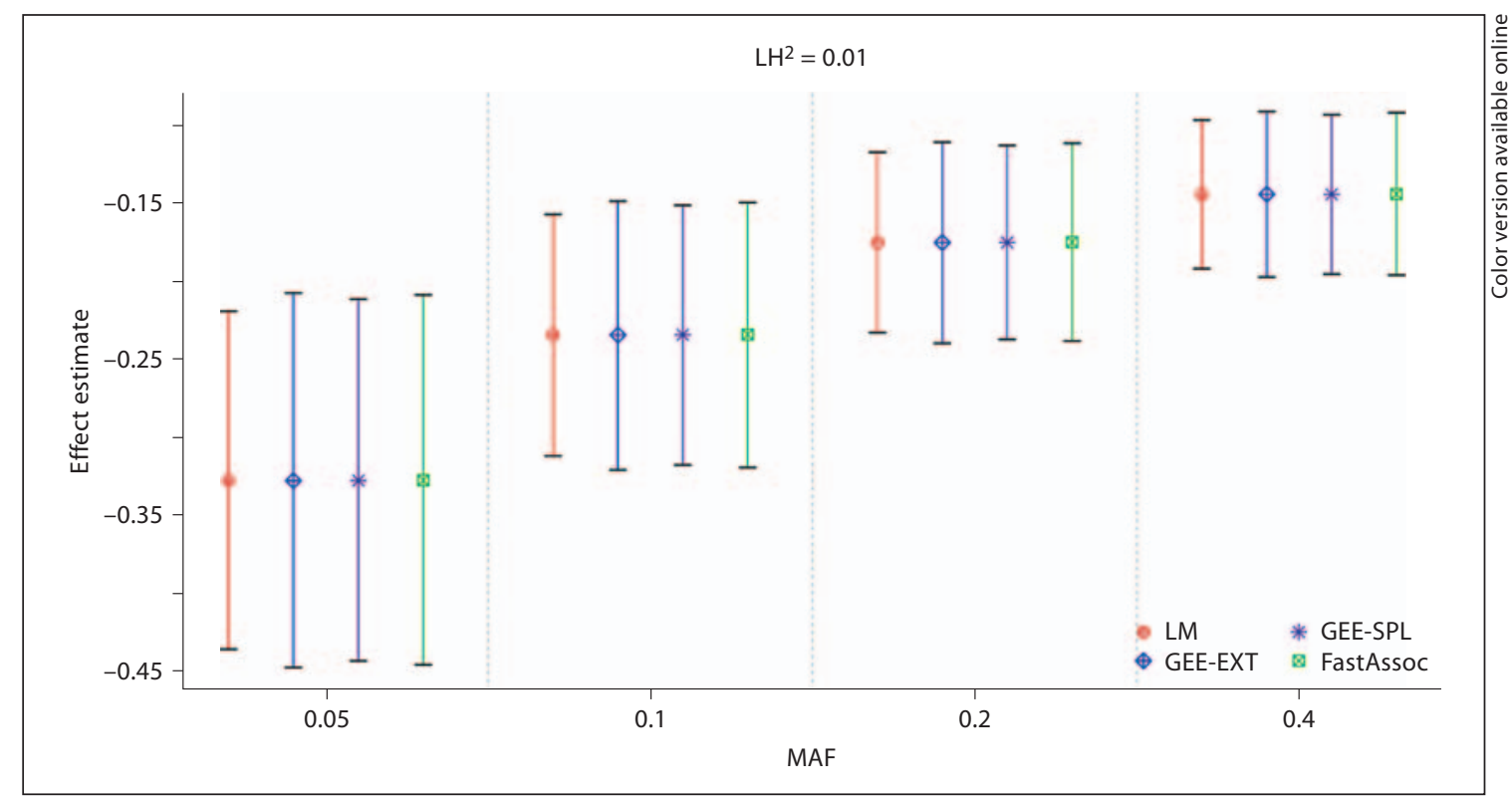

Fig. 3. Average effect size estimates and standard errors by association method across 10,000 replicates. The effect estimates are average effect estimates, and the error bars are the average standard errors across 10,000 replicates with a $1 \%$ locus-specific heritability at 4 different MAF. 


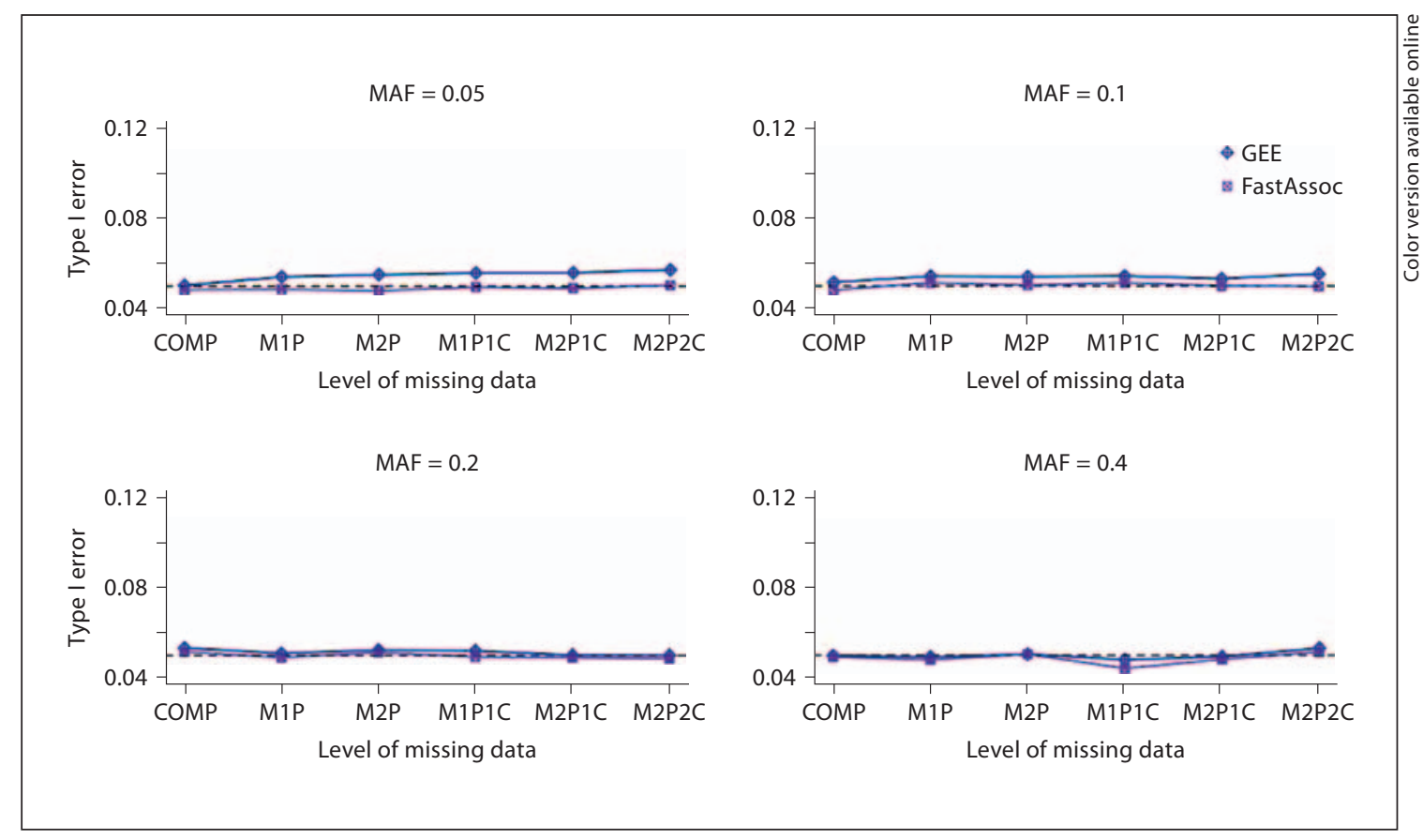

Fig. 4. Effect of missing data in nuclear families on type I error rates for GEE and FastAssoc. The $\mathrm{x}$-axis reflects 6 patterns of genotype missingness: $\mathrm{COMP}=$ complete data; $\mathrm{M} 1 \mathrm{P}=$ missing one parent; $\mathrm{M} 2 \mathrm{P}=$ missing both parents; $\mathrm{M} 1 \mathrm{P} 1 \mathrm{C}=$ missing one parent and one child; $\mathrm{M} 2 \mathrm{P} 1 \mathrm{C}=$ missing both parents and one child; $\mathrm{M} 2 \mathrm{P} 2 \mathrm{C}=$ missing both parents and children. The dashed line indicates a type I error rate of $5 \%$.

ilies. The two major concerns for this approach have been the inflated type I error rates and reduced power.

Here, we show that the power of GEE was very similar to that of VCA. VCA showed slightly more power than GEE when the locus-specific heritability was greater than $0.5 \%$; however, GEE showed a slight advantage over VCA when the locus-specific heritability was less than $0.5 \%$.

We confirmed the speculation that GEE applied to extended families would yield a slightly inflated type I error. Splitting extended families into smaller nuclear families to make the correlation structure within a family more closely resemble an exchangeable correlation structure was proposed to accommodate this type I error inflation. However, applying GEE on splits of extended families actually increased the type I error rate more than simply using GEE on the original extended family structure. This is most likely due to correlation across clusters (nuclear families that are related because they came from the same extended family). When type I error rates were calculated based on similar data sets with simulated independent nuclear families, this inflation disappeared. Although GEE can account for correlation among observations within each cluster, it still assumes independence between each cluster. As an aside, we compared our application of GEE-EXT (GEE applied to extended families data with a robust variance estimator) to the same approach without a robust variance estimator (GEE-NR), and saw an increase in type I error, highlighting the need for employing the robust variance estimator when applying GEE to extended pedigrees (online suppl. table 1).

For quantitative traits, a mixed effect model (MEM), i.e. a random intercept model, is the other alternative to GEE. However, to achieve the optimal type I error rate, it is necessary to specify the correct choice of the variancecovariance matrix for the mixed model. The choices of the variance-covariance matrix for MEM range from a simple exchangeable correlation structure to a matrix of kinship coefficients or an estimated IBD-sharing matrix. When an IBD-sharing matrix is used, MEM is equivalent to VCA methods. However, as previously noted, calculating the IBD-sharing matrix for every marker in the GWAS data is computationally intensive and is the major drawback of this option. To alleviate the complexity from using measured genetic data, the kinship coefficient, which is the expected average correlation between two relatives across all markers in the genome, can be used. However, this 
overall correlation is often different from the locus-specific IBD sharing, and thus using the kinship coefficient alone also does not always yield the correct standard error estimates (data not shown). In contrast to mixed models, GEE offers the convenient use of a simple exchangeable correlation structure. This simple correlation matrix together with the use of a robust variance estimator yields proper standard error estimates and a proper control for the type I error rate without computational burdens of VCA or MEM.

One limitation of our study was the limited number of simulation replicates performed, and thus the limit on $\mathrm{p}$ values and effect sizes that can be estimated for a given sample size. It is not feasible to perform thousands of simulations for GWAS-scale data sets. Thus, we chose no more than 10,000 replicates per simulation experiment, and evaluated type I error rates at three critical levels feasible for 10,000 replicates $(\alpha=0.001,0.01$, and 0.05$)$. We found a consistent pattern of comparison across the methods regardless of type I error rate evaluated and thus expect that our results hold when a GWAS threshold such as $5 \times 10^{-8}$ is applied.

As expected, the effect sizes were consistently estimated across all tested methods, although SE estimates for GEE of split families tended to be somewhat underestimated, consistent with the type I error results. Although the working correlation matrix is specified incorrectly for GEE on extended families, the use of the robust variance estimator provides appropriate type I error and coverage $[9,10]$. Thus, we recommend that the family structure remain intact, and that the GEE method can be applied to the original family structure to test for association using an exchangeable correlation structure. Although type I error rates for GEE in extended families were slightly higher for genetic markers with a low MAF $(<0.1)$, the type I error rate was appropriate when the genetic variants became more common in the sample.

Because extended families often do not contain complete genotype data for all family members, we also sought to ensure that missing genotypes did not affect our results regarding the appropriateness of using GEE methods in the family association context. Although we did not directly examine the effect of missing data in extended families, we did examine the relative impact of patterns of missing genotypes across methods among nuclear pedigrees. Our results showed that GEE performs similarly to VCA in the presence of variable degrees of missing data.

Ignoring correlations within families in LM doubled the type I error rate to almost $10 \%$ at a nominal significance level of 0.05 . However, we did not find that correct- ing for inflation of the type I error rate significantly reduced the power of association tests as has previously been reported when relatedness in family was ignored [20].

Another common method, implemented in FBAT/ PBAT, can be used for association studies in extended family data, but it does not use available phenotype information in the parents [3]. Hence, FBAT/PBAT often has limited power compared to VCA or GEE when phenotypes are available on all types of family members.

\section{Conclusion}

For genome-wide association analysis of family data, using a method such as GEE to screen for association signals can significantly reduce the amount of time needed to identify the potential signals in the genome. We showed that GEE, a faster alternative to VCA, works well in extended family structures without the need to split the families into nuclear families. For loci with a locus-specific heritability of at least 1\%, FastAssoc has a slight power advantage over GEE; however, the difference in power is minimal $(<2 \%)$. Thus, GWAS using GEE should not limit the ability to detect true associations and is only slightly more prone to false positives. Control of this slightly inflated type I error can be achieved by retesting the potential signals using the gold standard test with the proper type I error rate.

\section{Acknowledgements}

This work was supported in part by grants from the National Institutes of Health/National Heart, Lung, and Blood Institute grants U01 HL72518 and R01 HL087698 (R.A.M., L.R.Y., L.C.B., D.M.B.), Division of the Intramural Research of the National Human Genome Research Institute, NIH, the Department of Epidemiology, Johns Hopkins Bloomberg School of Public Health and Committee on Higher Education, and the Royal Thai Government scholarship (B.S.).

References

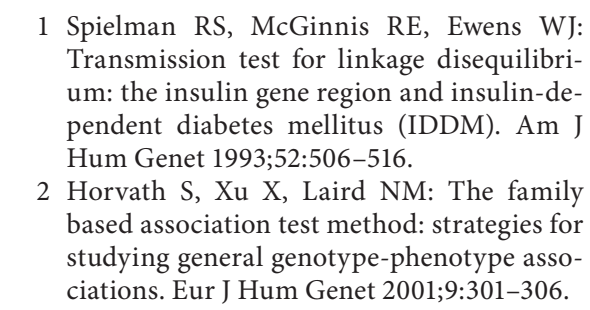

Hum Hered 2012;74:17-26 
3 Lange C, DeMeo D, Silverman EK, Weiss ST, Laird NM: PBAT: tools for family-based association studies. Am J Hum Genet 2004;74: 367-369.

4 Chen WM, Abecasis GR: Family-based association tests for genomewide association scans. Am J Hum Genet 2007;81:913-926.

$\checkmark 5$ Aulchenko YS, de Koning DJ, Haley C: Genomewide rapid association using mixed model and regression: A fast and simple method for genomewide pedigree-based quantitative trait loci association analysis. Genetics 2007;177:577-585.

6 Zhang Z, Ersoz E, Lai CQ, Todhunter RJ, Tiwari HK, Gore MA, Bradbury PJ, Yu J, Arnett DK, Ordovas JM, Buckler ES: Mixed linear model approach adapted for genomewide association studies. Nat Genet 2010;42 355-360.

$>7$ Kang HM, Zaitlen NA, Wade CM, Kirby A Heckerman D, Daly MJ, Eskin E: Efficient control of population structure in model organism association mapping. Genetics 2008; 178:1709-1723.

$\checkmark 8$ Kang HM, Sul JH, Service SK, Zaitlen NA, Kong SY, Freimer NB, Sabatti C, Eskin E: Variance component model to account for sample structure in genome-wide association studies. Nat Genet 2010;42:348-354.

$>9$ Liang K, Zeger SL: Longitudinal data analysis using generalized linear models. Biometrika 1986;73:13-22.
Zeger SL, Liang KY: Longitudinal data anal ysis for discrete and continuous outcomes. Biometrics 1986;42:121-130.

-11 Cupples LA, Arruda HT, Benjamin EJ, D’Agostino RB Sr, Demissie S, DeStefano AL, Dupuis J, Falls KM, Fox CS, Gottlieb DJ, Govindaraju DR, Guo CY, Heard-Costa NL, Hwang SJ, Kathiresan S, Kiel DP, Laramie JM, Larson MG, Levy D, Liu CY, Lunetta KL, Mailman MD, Manning AK, Meigs JB, Murabito JM, Newton-Cheh C, O'Connor GT, O’Donnell CJ, Pandey M, Seshadri S, Vasan RS, Wang ZY, Wilk JB, Wolf PA, Yang Q, Atwood LD: The Framingham Heart Study $100 \mathrm{~K}$ SNP genome-wide association study resource: overview of 17 phenotype working group reports. BMC Med Genet 2007;8(suppl 1):S1.

12 Tregouet DA, Ducimetiere P, Tiret L: Testing association between candidate-gene markers and phenotype in related individuals, by use of estimating equations. Am J Hum Genet 1997;61:189-199.

13 Vaidya D, Yanek LR, Moy TF, Pearson TA, Becker LC, Becker DM: Incidence of coronary artery disease in siblings of patients with premature coronary artery disease: 10 years of follow-up. Am J Cardiol 2007;100: 1410-1415.

14 R Development Core Team: R: A Language and Environment for Statistical Computing. Vienna, R Foundation for Statistical Computing, 2010.
15 Abecasis GR, Cherny SS, Cookson WO, Cardon LR: Merlin - rapid analysis of dense genetic maps using sparse gene flow trees. Nat Genet 2002;30:97-101

16 Kraja AT, Culverhouse R, Daw EW, Wu J, Van Brunt A, Province MA, Borecki IB: The Genetic Analysis Workshop 16 Problem 3: simulation of heritable longitudinal cardiovascular phenotypes based on actual genome-wide single-nucleotide polymorphisms in the Framingham Heart Study. BMC Proc 2009;3(suppl 7):S4.

17 Huber P: The behavior of maximum likelihood estimates under nonstandard conditions. Proc Fifth Berkeley Symp Math Stat Prob 1967;1:221-233.

18 White H: A heteroskedasticity-consistent covariance matrix estimator and a direct test for heteroskedasticity. Econometrica 1980; 48:817-838.

19 Vincent JC: GEE: generalized estimation equation solver, ported to R by Thomas Lumley (versions 3.13 and 4.4) and Brian Ripley (version 4.13). $\mathrm{R}$ package version 4.13-14. http://CRAN.R-project.org/package $=$ gee, 2009.

20 McArdle PF, O’Connell JR, Pollin TI, Baumgarten M, Shuldiner AR, Peyser PA, Mitchell BD: Accounting for relatedness in family based genetic association studies. Hum Hered 2007;64:234-242. 